\title{
Philosophiques
}

\section{Quelle juste part ? Normativité, remplaçabilité et portée}

\section{David Robichaud et Patrick Turmel}

Volume 41, numéro 1, printemps 2014

URI : https://id.erudit.org/iderudit/1025731ar

DOI : https://doi.org/10.7202/1025731ar

Aller au sommaire du numéro

Éditeur(s)

Société de philosophie du Québec

ISSN

0316-2923 (imprimé)

1492-1391 (numérique)

Découvrir la revue

Citer ce document

Robichaud, D. \& Turmel, P. (2014). Quelle juste part ? Normativité, remplaçabilité et portée. Philosophiques, 41(1), 177-193.

https://doi.org/10.7202/1025731ar d'utilisation que vous pouvez consulter en ligne.

https://apropos.erudit.org/fr/usagers/politique-dutilisation/ 


\title{
Quelle juste part? Normativité, remplaçabilité et portée
}

\author{
DAVID ROBICHAUD et PATRICK TURMEL
}

\section{Introduction}

Le cadre théorique de La juste part peut être associé à ce que l'on appelle en philosophie politique les positions « relationnelles» de la justice, ou «dépendantes de la pratique». Selon leurs tenants, aucun devoir moral ou obligation de justice ne précède les relations ou interactions sociales. Pour cette raison, toute réflexion sur la justice entretient une certaine relation de dépendance avec un ensemble de faits sociaux et politiques. Pour le dire autrement, les formes d'interaction, d'organisation et d'institutions qui font la vie sociale informent le développement et la justification des principes de justice. Il ne s'agit pas ainsi de soutenir que les formes de vie sociales sont, en soi, justes, voire de défendre le statu quo. Loin s'en faut. Seulement, les institutions et pratiques existantes ont un rôle à jouer dans la justification même de notre conception de la justice. Pour reprendre la définition que propose Andrea Sangiovanni de la thèse de "dépendance à la pratique ": "Le contenu, la portée et la justification d'une conception de la justice dépendent de la structure et de la forme des pratiques que cette conception est destinée à gouverner» (Sangiovanni, 2008, I38).

C'est la raison pour laquelle la thèse principale avancée dans l'ouvrage est que si les inégalités dont nous sommes aujourd'hui témoins doivent être en bonne partie corrigées, et donc qu'il existe une certaine obligation de justice en faveur de l'égalité, ce n'est pas à cause d'un devoir moral de stricte égalité qui existerait en amont de la vie sociale, dans le monde des idées. C'est plutôt une implication du fait que la coopération sociale dans une société démocratique qui refuse toute forme de hiérarchie naturelle entre les citoyens génère une double demande: d'abord, une demande de reconnaissance du rôle ou de la part de chacun à la production du "surplus coopératif », ensuite, une demande de justification de la légitimité de tout écart par rapport à une distribution égalitaire de ce surplus. Nous tentons ainsi de démontrer dans l'ouvrage que les inégalités actuelles - cet écart - ne reconnaissent pas correctement la part de chacun et que leur justification est insatisfaisante. Ainsi, comme nous l'expliquons dès l'introduction de l'ouvrage, "si les plus riches doivent payer davantage, contribuer plus que les autres dans les schèmes d'imposition et par la taxation, c'est d'abord et surtout parce qu'ils profitent davantage de la coopération sociale et des bénéfices collectifs produits » (Robichaud et Turmel 20I2, I I).

L'ouvrage soulève en conséquence plusieurs questions de fond en philosophie politique, et les trois auteurs qui interviennent dans ce dossier rappellent dans leur texte respectif trois des plus importantes parmi elles. 
Pierre-Yves Néron vient ainsi rappeler les problèmes associés au fait de défendre un principe comme l'égalité, à partir, notamment, de considérations empiriques contingentes. Peter Dietsch nous rappelle que les talents naturels ne justifient pas les écarts de revenus, mais que la remplaçabilité différentielle représente une justification morale des grands écarts de richesse qui est compatible, d'une part, avec le caractère moralement arbitraire des talents naturels et, d'autre part, avec l'importance de la coopération sociale dans la production de la richesse. Les individus ne méritent-ils pas de profiter des talents qu'ils ont choisis de développer? Enfin, Solange Chavel soulève un enjeu absolument crucial pour toute théorie de la justice aujourd'hui, qui est celui de l'échelle d'application des principes de justice. Compte tenu de l'importance normative que nous accordons dans La juste part aux relations d'interdépendance sociale, ne devrions-nous pas dépasser le cadre social (ou stato-national) qui est le nôtre pour appliquer notre réflexion et ses implications normatives au cadre global?

Nous remercions très chaleureusement nos collègues Solange Chavel, Peter Dietsch et Pierre-Yves Néron d'avoir si généreusement accepté de se prêter au jeu de la disputatio. Nous leur sommes reconnaissants de leur lecture attentive, et charitable. C'est un honneur et un plaisir d'être en discussion avec de si bons lecteurs.

\section{Réponse à Néron}

\section{De la normativité de la coopération sociale}

Dans son texte, Pierre-Yves Néron questionne l'apport de faits empiriques à la réflexion sur la justice, en rappelant d'abord l'utilisation qui est faite, dans La juste part, de travaux comme ceux des épidémiologistes Wilkinson et Pickett (2009), lesquels, dans leur monumental ouvrage The Spirit Level, dont les conclusions s'appuient sur l'étude des populations d'une vingtaine de pays développés, affirment que plus une société est inégalitaire, plus les problèmes sociaux (drogue, obésité, faible mobilité sociale, système d'éducation défaillant, etc.) sont importants. Ou, pour le dire autrement, le niveau d'inégalités dans une société donnée est le meilleur indicateur de la présence et de la gravité de ces problèmes. Ainsi, à peu près tous les problèmes sociaux associés aux groupes défavorisés sont davantage répandus et plus graves dans les sociétés les plus inégalitaires. Et ce, de façon très importante. La suite de l'étude a permis de conclure, en revanche, que plus un pays est égalitaire, mieux il excelle sur le plan de la santé et du bien-être de sa population générale. C'est ce que nous rappelons dans le dernier chapitre de notre essai pour affirmer que l'égalité profite à tout le monde, pas seulement aux moins nantis.

Tout en reconnaissant la force de persuasion que peut avoir ce genre de travail, Néron soulève deux craintes quant à l'utilisation de tels faits empiriques dans le cadre d'une théorie égalitariste, ou plus généralement d'une théorie normative. 
D'abord, qu'en serait-il si de nouvelles études remettaient en question les conclusions de Wilkinson et Pickett, voire qu'elles faisaient la preuve des méfaits de l'égalité? Faudrait-il alors abandonner nos intuitions égalitaristes?

Ensuite, et de façon plus fondamentale, l'appel aux considérations empiriques réduirait les considérations égalitaristes à une simple question instrumentale. Ainsi, comme l'explique Néron, affirmer que l'égalité est une bonne chose du point de vue du bonheur ou du bien-être a comme conséquence de la réduire à un simple moyen dans la recherche de biens qui, eux, possèdent une valeur intrinsèque. Il n'y aurait alors aucun argument pour justifier ou défendre l'égalité en elle-même. Pour le dire plus précisément, avec Néron, il n'y aurait pas d'obligation de justice à favoriser l'égalité. Un utilitariste strict pourrait par exemple être très à l'aise avec une telle conclusion: tant que l'égalité favorise l'utilité ou le bien-être global, elle doit être recherchée. Il est vrai qu'une part des arguments que nous avançons en faveur de l'égalité relève d'une telle logique instrumentale. Mais notre position présente aussi l'égalité comme une condition de la justice.

En fait, pour répondre à Néron, il est important de distinguer - ce qu'il fait par ailleurs dans son texte sans les nommer - trois types d'arguments avancés dans La juste part et qui dépendent au moins en partie de faits ou de contingences empiriques, mais qui se situent sur des plans normatifs différents. Appelons-les " argument du bien-être ", "argument de statut " et «argument de la coopération ".

Les craintes de Néron s'appliquent avec la plus grande force au type d'argument $d u$ bien-être: il a parfaitement raison d'affirmer que le genre d'argument selon lequel l'égalité profite à tout le monde - qu'elle favorise le bonheur ou le bien-être - n'est pas un argument normatif ou de justice. Cet argument est d'ailleurs bien périphérique à notre modèle normatif: il s'agit d'un "bonus » pour les sociétés égalitaires, voire d'une ultime tentative pour convaincre des individus parfaitement certains que les inégalités ne posent pas de problèmes, de justice ou autre. Pour établir un dialogue avec un égoïste rationnel, il peut être utile de mentionner que l'égalité profite à tous, même aux riches, même à lui. Il ne s'agit toutefois pas du cœur de notre argument en faveur de l'égalité.

L'argument de statut affirme quant à lui que les inégalités sont problématiques, parce que ce qui compte vraiment du point de vue de l'individu dans une société riche n'est pas son niveau de richesse absolu (que possèdet-il ?), mais sa situation socio-économique en comparaison de celle des autres (possède-t-il plus ou moins que les personnes qui évoluent dans son contexte de référence?).

Une intuition que semblent partager plusieurs personnes aujourd'hui est que ce ne sont pas les inégalités qui devraient d'abord nous préoccuper, mais le sort des moins favorisés. Il est d'ailleurs de bon ton aujourd'hui d'affirmer que les inégalités socio-économiques ne sont pas préoccupantes 
d'un point de vue moral, à la double condition que les plus riches aient acquis leur fortune par des voies légitimes et que nous offrions aux plus pauvres des conditions d'existence acceptables. Si ces deux conditions sont respectées, alors il n'y aurait rien d'injuste quant aux inégalités socioéconomiques qui en résultent.

En philosophie politique contemporaine, on parle du débat entre égalité et priorité. Ainsi, pour les égalitaristes, les inégalités, ou du moins certaines inégalités, sont en soi injustes, alors que pour les prioritaristes, ce qui importe est l'intérêt des plus démunis; c'est de leur sort que nous devons nous préoccuper en priorité. Pour certains, comme Derek Parfit ou Harry Frankfurt, les philosophes épris de justice ne devraient pas se préoccuper d'égalité à proprement parler, mais plutôt de savoir si les conditions de vie des individus sont suffisamment bonnes, dans l'absolu, et non pas relativement aux autres. L'argument du statut explique en partie pourquoi nous nous situons du côté des égalitaristes plutôt que du côté des prioritaristes.

Dans les pays « riches» ou développés, simplement avoir plus d'argent ou augmenter ses revenus ne semble pas avoir beaucoup d'effet sur le bonheur. Une fois que l'on a un foyer meublé, de la chaleur, de quoi se nourrir, et probablement aussi un téléphone, un téléviseur et toutes sortes d'autres babioles qui ne répondent sans doute pas aux besoins humains les plus fondamentaux, toute nouvelle possession risque d'avoir une incidence marginale superficielle sur notre bien-être. La satisfaction n'est pas liée au standard de vie absolu des individus, mais à la différence entre leur niveau de vie et celui des autres. Les individus tirent plus de satisfaction s'ils habitent une maison de 2000 pieds carrés dans un quartier où la maison moyenne offre I 200 pieds carrés que s'ils habitent une maison de 4000 pieds carrés dans un quartier où la maison moyenne offre 6000 pieds carrés. La richesse est une valeur toute relative. C'est toujours en se comparant aux autres que l'on détermine si l'on est riche ou pauvre, privilégié ou démuni. Et plus une société est inégalitaire, plus il y a de chance que des individus aient l'impression d'être floués, de ne pas avoir assez pour être heureux, ou qu'ils soient insatisfaits de leur situation. Est-ce la raison fondamentale pour favoriser l'égalité? Absolument pas. Il s'agit à nouveau d'un argument instrumental liant l'égalité à la satisfaction qui, encore une fois, nous permet de prendre la mesure de l'importance de l'égalité dans nos sociétés. En un mot, nos préoccupations pour le bonheur et le bien-être ne sont aucunement en contradiction ou en tension avec nos préoccupations de justice, mais elles en sont distinctes.

L'argument central de La juste part en faveur de l'égalité n'est donc ni l'argument de bien-être ni l'argument de statut, mais l'argument de la coopération. Pour comprendre comment la richesse doit être distribuée - et donc ce qui est une distribution juste -, il est essentiel de comprendre comment la richesse est produite - et donc qui a rendu possible cette production. La thèse centrale de La juste part est que toute richesse produite dépend 
de la coopération sociale, et qu'à l'inverse (presque) aucune richesse n'est le fruit du travail d'un individu isolé, qui n'aurait eu recours qu'à ses talents et vertus pour créer un bien d'une quelconque nature. C'est ce que nous avons tenté de démontrer par le fameux exemple du grille-pain.

Personne dans notre société ne peut, par ses propres connaissances et son savoir-faire, construire un grille-pain à partir de ressources brutes trouvées dans la nature. Pourtant, cet outil de tous les jours s'avère être d'une banalité désarmante lorsqu'on le compare à d'autres biens, tels les téléphones intelligents, les satellites qui les relient entre eux, ou un accélérateur de particules. Comment expliquer que des individus incapables de construire un grille-pain, à peine capables de se faire du pain, soient à même de parvenir, collectivement, à envoyer des robots sur Mars?

La réponse se trouve dans la coopération sociale. La production de toute richesse est un fait social, collectif. La contribution de chacun n'est jamais isolée, mais située à l'intérieur de relations et d'institutions sociales complexes et denses, et construite sur des acquis pour lesquels l'individu n'est pas responsable.

Si le talent joue un rôle - et il ne fait aucun doute que nous naissons inégaux quant aux talents, aptitudes et habiletés — nous avons tort de chercher à tout expliquer, succès comme échecs économiques, pour ce qui est du talent ou du mérite relatif des individus. En fait, trois autres facteurs ont sans doute plus d'importance que le talent ou le mérite.

Le premier de ces facteurs est la division du travail. La contribution de chacun n'a de valeur qu'en tant qu'elle s'insère de façon adéquate dans un système de division des tâches à accomplir. La division du travail permet la spécialisation dans des domaines plus ou moins pointus. Chacun ayant une tâche précise à accomplir, il est alors possible de s'améliorer et d'y exceller. Collectivement, nous arrivons à produire beaucoup plus que si tous partageaient leur temps entre la construction de maisons, la confection de vêtements, l'agriculture, l'élevage, la défense contre les ennemis et les animaux sauvages, etc. Ce qu'il faut garder à l'esprit, c'est que la production de richesses est un travail d'équipe: pendant que Bill Gates et Steve Jobs s'affairaient à changer nos vies, une armée d'individus veillaient à ce qu'ils aient accès à des ressources alimentaires, à ce que leur habitation soit chauffée, à leur sécurité et celle de leurs enfants, à l'éducation de ces derniers, etc. C'est parce que certains acceptent de consacrer leur vie à l'agriculture, à l'enseignement, à la garde d'enfants et à de nombreuses tâches différentes que d'autres peuvent consacrer la leur à la médecine, à la finance ou au développement de produits informatiques.

Le second facteur est l'héritage matériel et immatériel. Personne ne commence sa vie nu sur une île déserte. Quand nous naissons, nous avons accès à des biens qui représentent un patrimoine gigantesque, légué par les générations passées. Ce patrimoine est composé de biens matériels, comme des routes, des ponts, des hôpitaux, des barrages hydroélectriques, des bri- 
quets, etc. Il se compose aussi de biens immatériels. La culture, la connaissance accumulée et archivée sont ici des exemples évidents. La contribution de chaque individu se fait donc toujours à partir des acquis et du travail réalisé par ceux qui l'ont précédé. Chaque avancement réalisé par un individu n'est possible que parce que d'autres ont mis en place les conditions de possibilité de cet avancement, et rendu accessible ce patrimoine.

Le troisième facteur, qui aurait pu être inclus dans le patrimoine immatériel mais qui mérite d'être présenté indépendamment pour en illustrer l'importance, c'est ce que nous pouvons appeler le patrimoine normatif des collectivités dans lesquelles nous évoluons et interagissons. Notre vie collective est régie par un ensemble de règles et de normes sans lesquelles la vie sociale en général et la vie économique en particulier seraient impossibles. Nous conduisons à droite, nous ne pouvons nous approprier un objet qui est la propriété d'autrui sans son accord, nous adoptons un horaire de travail distinct les fins de semaine, nous nous habillons en noir aux enterrements, nous ne nous marions pas entre frères et sœurs, etc. Toutes ces règles, mais surtout le fait que la vaste majorité des individus s'y soumettent, rendent possibles non seulement une vie collective harmonieuse, mais la production de richesses.

Le simple fait que je respecte les normes régissant la propriété fait de moi un contribuable à l'entreprise de coopération. Si un individu peut se consacrer au développement de compétences lui permettant de diriger une entreprise, c'est parce qu'il a l'assurance qu'il pourra profiter des bénéfices de cet investissement ou de cette prise de risques. Sans la contribution de chacun au maintien de ce patrimoine normatif, la production de richesses dans nos sociétés serait sinon impossible, du moins sans grand résultat.

Si le mérite ou le talent individuel joue un certain rôle dans la détermination du succès individuel, c'est donc surtout l'accès aux occasions offertes par la coopération sociale qui, en nous permettant de développer certains talents, influence le plus nos chances de succès ou d'échecs économiques. Si tous ne naissent pas avec les mêmes talents, tous n'ont pas non plus un accès égal aux possibilités et aux ressources produites par la coopération, et cela pose problème du point de vue de la justice.

L'argument de la coopération se présente donc ainsi: quiconque produit un bien sans l'aide de qui que ce soit est le propriétaire légitime de ce bien et ne doit rien à personne. Robinson Crusoé, seul sur son île, qui construit une hutte, cultive un potager et élève quelques bêtes, est en principe unique propriétaire de ces biens. Si quelqu'un fait naufrage et échoue sur l'île de Robinson, ce dernier a peut-être un devoir d'entraide, mais pas d'obligation de justice envers le nouvel arrivant.

Par contre, le corrolaire de cette idée est que quiconque participe à la création d'un bien a droit à sa juste part. Et dès lors que nous prenons la mesure de l'apport de la coopération sociale à toute forme de contribution dans la production de richesse, il devient rapidement évident que cette pro- 
duction n'est jamais le fruit d'individus isolés, mais toujours celui d'une entreprise collective, à laquelle chacun contribue (de façon inégale, mais tout de même difficilement quantifiable). Tous ne profitent toutefois pas à la même hauteur du schème de division du travail et des produits du patrimoine collectif. Certains, en raison sans doute de qualités naturelles, mais surtout grâce aux aléas de la vie, ont développé des compétences et des talents qui leur permettent d'atteindre des positions sociales privilégiées. À l'opposé, des individus n'ont pas eu autant de chance et se retrouvent dans des positions sociales moins enviables.

Ce qui est à retenir ici est: I) que la contribution de la majorité d'entre nous est nécessaire à la coopération sociale telle que nous la connaissons; 2) que les contributions individuelles sont beaucoup plus égales que l'on a tendance à l'imaginer. La distribution de la richesse devrait refléter cette réalité. De ce point de vue, les seules inégalités légitimes sont celles qui découlent du travail parfaitement isolé d'un individu - ce qui se réduit à bien peu de choses - ou celles qui sont nécessaires pour motiver les individus les plus compétents à mettre leurs talents et savoirs au service de la production de biens ou services collectivement désirables ou désirés - nous verrons dans la prochaine section que l'argument de la motivation ne justifie sans doute pas non plus les importantes inégalités actuelles.

Selon l'argument de la coopération, la personne qui est dans une situation socio-économique privilégiée doit donc son sort en bonne partie au fait d'avoir profité davantage que les autres de l'entreprise de coopération qu'est la société. Pour nous, cela signifie que la personne socio-économiquement favorisée est d'une certaine façon dans une position de dette envers une société sans laquelle sa réussite et son enrichissement auraient été impossibles. Par exemple, laisser s'enrichir la personne jugée compétente revient à la récompenser doublement: elle profite davantage des fruits de la coopération dans le développement de ses talents, puis elle fait jouer cet avantage concurrentiel à son bénéfice sur le marché économique. C'est ce que doit corriger la redistribution de la richesse. D'où la justification proposée en faveur d'une plus grande redistribution de la richesse. Sans qu'il soit pleinement normatif - si l'on entend par là qu'il ne dépend d'aucune façon de contingences sociales ou institutionnelles — c'est ici l'argument principal de La juste part, et nous croyons qu'il s'agit du meilleur argument en faveur de la justice distributive.

\section{Réponse à Dietsch}

\section{De la remplaçabilité des individus}

Peter Dietsch nous presse de clarifier notre position sur la légitimité des salaires élevés des chefs d'entreprises. Nous questionnons dans l'ouvrage la justice de la rémunération de ces derniers, pouvant atteindre plusieurs centaines de fois le revenu de certains de leurs employés. En mettant l'accent sur l'apport de la société à la réussite de certains individus, et en rejetant l'argu- 
ment du mérite individuel, du talent et des vertus de l'individu dans l'explication de son succès social et économique, nous cherchons à rendre moralement suspecte la compensation financière qu'ils touchent.

Dietsch mentionne le cas intéressant de Jack Welsch, PDG de General Electric de I98 I à 2000, et nommé Gestionnaire du siècle par le magazine Fortune en 1999. Selon Dietsch, ce ne sont pas les qualités naturelles de Welch, ses vertus ou ses talents, qui en font un individu méritant de telles compensations financières, mais bien ses compétences acquises et la difficulté de le remplacer. Sa formation, son savoir gestionnaire, mais aussi sa connaissance particulière de l'entreprise pour laquelle il travaille, ici GE, en feraient un individu irremplaçable. Peter Dietsch nous invite donc à réfléchir sur la possibilité d'accepter l'idée que les talents naturels n'expliquent pas les contributions individuelles et ne justifient pas les inégalités salariales que nous connaissons. Selon lui, la contribution de chacun est donc dépendante de l'existence d'un patrimoine matériel, immatériel et normatif, mais, malgré tout, les compétences et connaissances acquises pendant une vie justifient des inégalités salariales en raison de la difficulté relative de remplacer certains "producteurs de richesse".

L'auteur nous offre donc l'occasion de clarifier notre position et d'expliquer pourquoi nous rejetons la thèse intuitive de la remplaçabilité comme justification des compensations offertes aux chefs d'entreprises. Pour présenter notre position rapidement, disons que les salaires élevés ne sont pas justifiés moralement par la difficulté relative de remplacer un individu, mais plutôt par la motivation qu'ils créent, chez les individus se trouvant dans la meilleure position sociale, de développer des compétences de haut niveau. Investir dans l'acquisition de compétences très spécialisées représente un grand risque pour l'individu, et comme ces compétences sont très productives collectivement, il est nécessaire de rendre ces investissements individuels attrayants. Ces incitatifs visent à produire, chez certains individus, une motivation telle qu'ils développeront des compétences exceptionnellement pointues, ce qui, par voie de conséquence, les rendra très difficiles à remplacer. Cette difficulté de les remplacer s'avère par contre être un effet des salaires inégaux, et non leur justification.

Pour défendre notre position, nous commencerons par distinguer la théorie de la remplaçabilité comme théorie explicative et comme théorie justificative, puis nous tenterons de démontrer que, s'il s'agit d'une théorie explicative, d'une grande pertinence, elle n'arrive à justifier ni économiquement ni moralement les inégalités créées par les rémunérations des chefs d'entreprises.

Explication ou justification? Il ne fait aucun doute dans notre esprit que la meilleure façon d'expliquer la rémunération d'un individu dans notre société est d'évaluer la difficulté de le remplacer ou la rareté des compétences qui sont les siennes - des compétences plus rares (et en demande) étant associées à des rémunérations plus substantielles. Les compétences, 
comme tout autre type de bien, sont soumises à la loi de l'offre et de la demande, et des compétences rares très en demande confèrent à leur propriétaire un rapport de force permettant de faire monter les enchères.

Problème de la justification économique. Il est possible d'imaginer une justification économique des compensations offertes aux chefs d'entreprises. Si les compétences d'un individu permettent de maximiser les investissements des actionnaires d'une entreprise, il est légitime d'offrir une compensation qui convaincra cet individu de mettre ses compétences uniques au service de l'entreprise, quelles que soient leur origine ou le mérite de l'individu à les posséder. Jack Welch, le PDG de GE qui exemplifie le propos de Dietsch, possédait certes des compétences uniques. Ses méthodes de gestion, sa connaissance du marché et sa connaissance de l'entreprise qu'il dirigeait en faisaient un individu extrêmement difficile à remplacer, pour ne pas dire irremplaçable. Cette situation explique sans doute qu'il ait pu négocier une prime de départ de 4I 7 millions. Somme énorme, qui fit d'ailleurs ternir son étoile de super-vedette de la gestion, même en considérant que la valeur de GE avait augmenté de 4000 p. cent pendant sa présidence.

Tournons-nous maintenant vers le numéro 2 de GE à l'époque. Sous Jack Welch se trouvait celui que tous voyaient comme son successeur éventuel: Gary Wendt. Il dirigeait GE Capital, la division la plus rentable de GE, responsable de $40 \mathrm{p}$. cent des profits de l'entreprise en I 998 , au moment où Wendt quitte ses fonctions. Après une tentative infructueuse de vivre une vie paisible à la campagne, loin des milieux financiers, Wendt ne put résister à l'offre de Conseco Inc., une compagnie dont les domaines de spécialisation étaient les siens: la finance et l'assurance. Une offre comprenant une compensation de près de deux cents millions de dollars et un bonus de signature de quarante-cinq millions est difficile à refuser. Ces conditions de travail généreuses peuvent s'expliquer par le fait que Wendt était vu comme le meilleur gestionnaire qui n'était pas déjà chef d'une entreprise. Il était donc, par ses compétences et son talent, d'une extrême rareté sur le marché. La fin de l'histoire est par contre très différente de celle de Welch. En effet, loin d'avoir fait opérer sa magie chez Conseco Inc., Gary Wendt démissionna plutôt, en octobre 2002, trois mois avant que la compagnie ne déclare faillite. Ce sont les mêmes connaissances et compétences qui avaient motivé l'octroi de sommes astronomiques à "Gary le gambler ", les mêmes connaissances et compétences qui avaient fait sa renommée chez GE Capital, les mêmes connaissances et compétences qui le rendaient extrêmement difficile à remplacer.

Il faut noter qu'il ne s'agit pas d'une anomalie rare et que le cas de Wendt n'est pas unique: il n'existe pas de corrélation entre les compensations offertes aux PDG et leur performance au sein d'une entreprise. Ce qui rend intuitivement acceptable de récompenser les individus en fonction de la rareté de leurs compétences, c'est que nous associons compétences à performances, et concluons que ces performances justifient une compensation. Le 
lien entre compétences et performances est malheureusement beaucoup moins direct qu'on ne le suppose. Dans les années I990, période où les compensations offertes aux PDG ont explosé, «les gestionnaires ont fait jouer leur influence pour obtenir des compensations plus importantes en privilégiant des arrangements salariaux fortement indépendants des performances. [...] La rémunération des dirigeants est beaucoup moins liée à la performance qu'on ne le croit généralement» (Bebchuk \& Fried, 2004, 6).

Quelques exemples? Deux tiers des fusions s'avèrent de mauvaises décisions de la part des PDG, $80 \mathrm{p}$. cent des nouveaux produits ne survivent pas à leur première année sur le marché, et les marges de profit des entreprises n'ont pas augmenté dans les années 1990 alors que les revenus des PDG des grandes entreprises ont explosé, atteignant plusieurs centaines de fois le salaire du travailleur moyen. Continuons. Une étude menée sur vingt ans et analysant les vingt-cinq chefs d'entreprises les mieux rémunérés aux États-Unis pour chacune de ces années montre que près de $40 \mathrm{p}$. cent d'entre eux ont vu leur entreprise ( $22 \mathrm{p}$. cent) fermer ses portes ou être renflouée par des fonds publics afin d'éviter la faillite; ou bien ( 8 p. cent) ont été congédiés; ou encore étaient à la tête d'entreprises $(8$ p. cent) qui ont été accusées de fraude et ont dû payer des sommes importantes en amendes ou en règlements de toute sorte (IPS 20I3).

À partir du moment où les compétences d'un individu ne permettent pas de prédire son succès relatif comme dirigeant d'entreprise, il devient assez difficile de comprendre la rationalité de la rémunération sur la base de la difficulté de remplacer les compétences particulières d'un individu. La justification économique semble donc plutôt ténue pour légitimer des inégalités de l'ordre de celles que nous connaissons.

Évidemment, on pourrait toujours avancer que d'autres candidats moins compétents auraient fait encore pire. Cela est peu convaincant comme argument pour réhabiliter la théorie de la remplaçabilité comme théorie justificative d'un point de vue économique.

D'abord, la différence de compétences entre les PDG et les aspirants à ces postes est beaucoup plus mince que ne l'est la différence entre leur salaire respectif. Robert Frank et Philip Cook ont d'ailleurs bien rappelé, dans The Winner-Take-All Society (1995), la façon dont furent mises en place des structures de rémunération qui offrent des prix de plus en plus alléchants aux "premiers de classe ». Des compétences ne serait-ce que très légèrement supérieures à celles d'autrui se traduisent sur certains marchés par des inégalités salariales ahurissantes. Dans des domaines très concurrentiels où plusieurs individus très talentueux et très motivés sont en compétition, les meilleurs n'ont parfois qu'une supériorité infime sur les seconds de classe (pensons aux Olympiques), mais cette supériorité se traduit néanmoins par des gains très inégaux. Ce que montrent Frank et Cook est que ce phénomène ne cesse de prendre de l'ampleur, en termes de domaines concernés et en termes des écarts salariaux produits, notamment à cause d'un marché qui 
connaît de moins en moins de frontières. Il explique notamment l'énorme différence de revenu entre le chef d'entreprise et ses seconds.

Ensuite, il est loin d'être clair, dans un contexte d'incertitude et d'impondérables, que la légère supériorité quant aux compétences représente une quelconque assurance de performance supérieure. Finalement, et de façon plus évidente, n'y a-t-il pas de limite à la prime salariale que peut procurer une légère supériorité en matière de compétences? L'argument de la remplaçabilité ne nous dit rien sur le sujet, et s'il semble légitime et justifié que les meilleurs reçoivent davantage que les autres, il semble aussi nécessaire de disposer d'un moyen de mettre fin à la flambée des salaires. Un individu irremplaçable mérite-t-il un salaire illimité?

La seule justification économique acceptable, à notre avis, est liée à la motivation: les salaires élevés sont justifiés dans la mesure où ils motivent des individus à rivaliser pour les obtenir et ainsi maximiser la production de richesse collective. Â partir du moment où la motivation n'est plus influencée positivement, qu'elle demeure neutre ou est influencée négativement par la rémunération marginale (Pink, 2009), nous avons atteint le point maximal de compensation justifiable économiquement. Chaque dollar supplémentaire octroyé aux PDG est une perte nette pour les actionnaires, ou joue contre l'intérêt des actionnaires et de la société en général. La thèse de la remplaçabilité ne s'intéresse pas à la rémunération comme élément motivateur, mais comme récompense de l'acquisition d'avantages concurrentiels, sans lien nécessaire avec la richesse que ces compétences permettent de produire.

Le problème de la justification morale. Le troisième argument à propos de la compensation des PDG est de nature morale. En plus d'être empiriquement discutables et inefficaces, de très importantes compensations pour les chefs d'entreprises posent problème du point de vue de la justice. Dietsch concède que les talents naturels ne permettent pas de comprendre les rémunérations qu'obtiennent les dirigeants des grandes entreprises, mais leurs compétences supérieures, elles, le permettraient. Pour répondre à cette critique, nous devons montrer non seulement que les talents sont moralement arbitraires, mais qu'une bonne partie de leur développement l'est aussi.

Nous avons tenté dans La juste part de démontrer que le développement des compétences dépend non seulement de facteurs qui dépassent la volonté et la responsabilité individuelle, mais aussi de ressources rendues disponibles par la coopération sociale, à laquelle participe la vaste majorité. La coopération sociale offre à certains les moyens de devenir irremplaçables et un contexte dans lequel nous avons la possibilité de profiter de ce statut privilégié.

Rappelons que, sans la division du travail et sans le savoir accumulé par les générations passées, il serait impensable de développer des compétences extrêmement précises et ciblées. Or ce sont ces compétences qui aujourd'hui permettent d'obtenir des gains économiques importants sur le 
marché. Une culture, des croyances et des coutumes nous sont aussi transmises, qui influencent grandement nos choix de carrière ou les talents que nous choisissons de développer. Les modèles sociaux associés aux genres ou à l'origine géographique des individus viennent ici à l'esprit. Finalement, rappelons que sans un patrimoine normatif partagé et très généralement respecté, nos incitatifs à investir dans l'acquisition de compétences seraient beaucoup plus faibles. Nous devons aussi garder à l'esprit que certaines règles adoptées socialement créent des avantages pour certains individus, comme nous le rappelle l'exemple des cohortes scolaires discuté dans $\mathrm{La}$ juste part (Robichaud et Turmel 201 2, 65).

De là, nous concluons que les salaires astronomiques octroyés à certains dirigeants d'entreprise et à certaines personnes occupant des positions sociales privilégiées ne peuvent être justifiés par la difficulté de remplacer ceux qui possèdent les compétences requises, puisque ces compétences ne relèvent pas entièrement de leur mérite moral individuel. En fait, ils doivent le développement de leurs compétences aux conditions offertes par la coopération sociale.

Nous devons éviter de récompenser la chance et le hasard, et nous devons éviter de récompenser doublement les individus qui ont bénéficié plus que d'autres des ressources offertes par la coopération sociale et qui ont déjà la chance d'occuper des positions sociales enviables. C'est malheureusement ce que font ceux qui justifient les salaires en fonction de la remplaçabilité des individus.

Cela ne signifie donc pas qu'aucune inégalité n'est justifiée. Les inégalités peuvent jouer un rôle central dans la motivation des individus à développer leurs talents et à orienter ces talents dans les domaines qui nous semblent, collectivement, les plus importants. La justification morale des inégalités est ex ante et non ex post: les salaires élevés offerts aux meilleurs de chaque domaine sont justifiés par l'importance sociale de ces domaines, et ce sans connaître l'excellence et les compétences des vainqueurs. En contrepartie, si la concurrence permet d'améliorer la compétence des candidats, elle les rend aussi, éventuellement, plus difficiles à remplacer. Les récompenses doivent certes être suffisamment élevées pour motiver les plus talentueux à entrer en concurrence pour les obtenir, mais elles ne devraient pas dépasser le seuil à partir duquel elles ne jouent plus aucun rôle sur le plan motivationnel. D'ailleurs, selon Bebchuk et Fried, au cours des années 1990, les entreprises auraient pu produire les mêmes incitatifs de performance à de bien moindres coûts, ou auraient pu utiliser les sommes versées pour produire des incitatifs de performance beaucoup plus efficaces (Bebchuk \& Fried, 2004).

\section{Réponse à Chavel}

\section{De la portée de la juste part}

Solange Chavel soulève une difficulté de taille pour toute théorie de la justice sociale, mais qui s'avère particulièrement sérieuse pour la nôtre: celle de 
savoir comment justifier l'inscription de notre réflexion sur la justice dans les limites de l'échelle sociale ou stato-nationale, en considération de la thèse avancée du patrimoine coopératif, selon laquelle toute richesse est le produit d'un système de coopération qui a permis la constitution non seulement d'un patrimoine matériel, mais également d'un patrimoine normatif, et dont l'implication première est de mettre à mal nos représentations courantes de la justice qui attribuent un poids exagéré au mérite individuel.

Comme l'explique Chavel, la thèse du patrimoine coopératif ou des réseaux d'interdépendance devrait nous amener à remettre en question l'asymétrie entre le national et le global, laquelle justifierait une discussion de la justice sociale qui serait faite en vase clos, hors de toutes considérations internationales ou globales. En d'autres mots, si le cœur de l'argument porte sur l'interdépendance, on ne peut ignorer aujourd'hui que l'interdépendance sociale ne s'arrête pas aux frontières nationales. Peut-on dans ce contexte, demande Chavel, "limiter l'analyse de la justice sociale aux rapports entre les citoyens?".

Chavel offre des raisons pragmatiques pour justifier ce choix: "Nos mécanismes de redistribution des richesses ou de solidarité opèrent à l'échelle nationale; nos institutions politiques - et donc la sphère de débat public — sont encore essentiellement nationales; les chiffres, données et analyses dont nous disposons adoptent elles aussi cette même lentille. » À l'opposé, le manque criant de structures institutionnelles à l'échelle globale semble rendre impraticable l'application d'une théorie de la justice égalitariste ou distributive. Peut-être devrions-nous alors nous contenter de réfléchir aux conditions de justice sociale ou nationale et de présumer un minima de justice beaucoup plus bas à l'échelle globale, lequel se limiterait au respect des accords internationaux, et en premier lieu des accords de commerce «médiés par les organisations internationales"?

Mais il y a un autre problème, souligne Chavel, c'est que le genre de mécanismes que semble exiger la justice à l'échelle nationale - l'exemple offert en conclusion du livre est celui d'une augmentation du taux marginal d'imposition dans les tranches supérieures de revenu — ne peut être institué en vase clos, en ignorant les modes d'organisation économiques d'autres pays, sans par ailleurs produire de sérieux effets pervers. Au fond, ce que rappelle Chavel, c'est que les problèmes d'action collective du genre dilemme du prisonnier, dont on donne de nombreux exemples dans l'ouvrage, existent et sont même plus importants entre les États qu'entre citoyens à l'intérieur des frontières. C'est le cas lorsqu'il est question de régime fiscal — comment augmenter la charge fiscale des contribuables sans inciter ceux-ci à se déplacer dans une juridiction moins exigeante de ce point de vue? - mais aussi de règlementations environnementales, de normes du travail, de barrières tarifaires, parmi maints exemples. Nous ne pouvons qu'admettre la prégnance de ce genre de problèmes de coopération à l'échelle globale. Selon Chavel, l'implication, et donc le défi, est d'offrir un plaidoyer égalitariste mondial. 
Si nous sommes en accord avec Chavel lorsqu'elle affirme, compte tenu de l'importante interdépendance, qu'il est impératif aujourd'hui de "travailler à l'élaboration d'un sentiment non national de justice", nous croyons toutefois que le développement de ce sentiment non national de justice doit être distingué de l'application d'une théorie de la justice distributive "à l'échelle supérieure ", c'est-à-dire, à l'échelle globale. Au fond, la question est de savoir s'il n'existe pas tout de même des raisons de principe pour justifier une asymétrie entre le national et le global. Pour Chavel, l'exemple du problème de coopération fiscale démontre "qu'il est en fait illusoire d'enfermer le débat sur la justice distributive à l'intérieur de nos frontières nationales". Nous croyons plutôt que, s'il existe de bonnes raisons justifiant une réflexion sur la justice globale - notamment l'argument de l'interdépendance - les demandes de justice ne sont pas les mêmes à ces deux échelles.

Comme nous l'avons souligné dans l'introduction, le cadre qui est le nôtre dans l'ouvrage - prenant le parti de Hobbes contre Locke - nous situe clairement du côté du «relationalisme» ou de la «dépendance à la pratique» en philosophie politique. Pour nous, les principes de justice ne précèdent pas les institutions et ne sont pas entièrement extérieurs à cellesci. Ce sont des principes dont le contenu est déterminé par la structure de la coopération sociale. Cette structure de la coopération sociale altère fondamentalement les relations dans lesquelles les individus se trouvent, et donc les principes de justice qui sont appropriés.

Si ce cadre permet généralement aux auteurs qui s'en réclament de justifier une limitation des questions de justice au cadre stato-national, rien n'empêche par ailleurs un «institutionnaliste » d'insister justement sur l'importance des formes d'interaction et de coopération qui existent et continuent de se développer par-delà les frontières. Mais la reconnaissance de cette réalité n'implique pas nécessairement que nous appliquions les mêmes principes de justice à l'échelle sociale et à l'échelle globale.

La raison pour laquelle nous nous concentrons sur les institutions nationales n'est donc pas banalement pragmatique. Elle découle de la reconnaissance que les principes de justice sociale sont indissociables de toute l'épaisseur de la coopération à l'échelle nationale. En ce qui nous concerne, les institutions sociales de base, opérateurs de la coopération sociale à l'échelle nationale, règlent de la manière la plus fondamentale la vie des individus qui y prennent part - qui vivent sur le territoire particulier contrôlé par cette structure institutionnelle - en leur imposant un cadre normatif dans lequel ils doivent agir: un ensemble de règles et de contraintes auxquelles ils ne peuvent complètement échapper, à partir desquelles est déterminé ce qu'ils peuvent ou ne peuvent pas faire, et comment ils peuvent le faire. Il n'est pas ici simplement question du cadre juridique imposé par le droit. Cette structure comprend l'ensemble des institutions sociales qui déterminent les droits et les devoirs fondamentaux de l'individu, ainsi que 
les mécanismes par lesquels sont alloués les bénéfices et les fardeaux de la coopération sociale, ce qui concerne le système légal, bien sûr, mais aussi la constitution et le fonctionnement du système économique.

Ces institutions déterminent toute position sociale que peut occuper un individu, la façon dont ce dernier peut en venir à l'occuper ou à la perdre, la façon dont il peut s'approprier des ressources ou les échanger, établir des relations contractuelles particulières, voire même où et comment l'individu peut se déplacer, occuper ou utiliser l'espace. Par exemple, mon droit de posséder telle ou telle chose particulière dépend non seulement de la propriété privée, qui dépend à son tour d'un cadre institutionnel, mais aussi du fait que j'ai respecté les règles suivant lesquelles je pouvais m'approprier cette chose. De même en ce qui concerne mon rapport à l'espace, aux lieux qui me sont accessibles et à ceux qui me sont interdits. Tout cela dépend, en dernière instance, des institutions nationales. C'est ce que Rawls entend lorsqu'il affirme à propos de la structure de base qu'elle est le cadre dans lequel «se situe la poursuite de toutes les autres activités» (Rawls I997, 272; voir aussi Hodgson 2012 pour le détail de cette interprétation de Rawls). La structure de base n'est pas qu'un obstacle à l'agir parmi d'autres, elle est l'ensemble des règles et des contraintes que je dois respecter pour agir et pour poursuivre mon plan de vie. Elle contraint donc ainsi mon comportement, mais plus fondamentalement aussi le type de fins que je peux poursuivre. La légitimité de ces institutions exige donc que nous formulions des règles qui paraîtront raisonnables aux yeux de tous les coopérants. C'est aussi ce qui justifie un traitement égal de chacun de ses membres.

En comparaison, s'il y a interdépendance à l'échelle globale, il n'y a évidemment pas le même degré de coopération. En fait, on peut distinguer trois formes distinctes de "coopération".

Il y a d'abord ce que l'on a appelé dans La juste part la "coordination ", qui représente des formes d'interactions mutuellement avantageuses et auxquelles personne n'a de raison de résister une fois que l'on s'y est engagé. L'exemple classique, utilisé dans l'ouvrage, est le côté de la route sur lequel doivent conduire les automobilistes. Il y a peu de raisons de préférer une option (conduire à gauche) à une autre (conduire à droite). L'important, c'est que tout le monde respecte la même convention. Il ne s'agit pas seulement d'une forme de coopération profitable pour tous; une fois que l'on s'est entendu sur le contenu de la convention, personne n'a de raison de la violer.

Il y a ensuite ce que l'on a appelé la coopération en tant que telle. Il s'agit alors d'une forme d'interaction profitable pour tous, mais qui demeure plus difficile à faire respecter, simplement parce que, même s'il s'agit d'interactions mutuellement avantageuses, les participants ont toujours intérêt à faire cavalier seul, c'est-à-dire à préférer ne pas contribuer à la coopération et à profiter des bénéfices produits par autrui. C'est le fameux problème de l'insensé chez Hobbes, devenu le problème du resquilleur ou du free-rider 
dans la littérature contemporaine. Il serait mutuellement avantageux que tous prennent les transports en commun - pour éliminer les problèmes de congestion et améliorer la qualité de l'air - mais si tous les autres optent pour le transport public, il devient très avantageux de se déplacer en automobile sur des routes dégagées, pour un effet indiscernable quant à la qualité de l'air.

Mais il y a une troisième forme de "coopération ", que l'on discute aussi sans la nommer comme telle: la redistribution. Il s'agit d'une forme de coopération qui permet de réaliser certains idéaux de justice, mais plus généralement de faire face, de façon solidaire, à certains risques. Le filet social participe de cette logique. Nous acceptons de mettre une partie de nos ressources en commun pour aider ceux parmi nous qui auront la malchance de traverser une mauvaise passe. C'est aussi le cas de l'assurance-santé. Les dépenses de santé, contrairement à la plupart de nos autres dépenses courantes, sont complètement imprévisibles. Nous n'aurons pas tous besoin de la même quantité de soins de santé au cours de notre vie. Certains d'entre nous vivront plus vieux, d'autres mourront bien plus jeunes, certains auront à subir d'importantes interventions ou des soins à long terme, d'autres passeront leur vie sans même être hospitalisés une nuit. Mais personne ne sait quelle sera sa situation propre. Impossible, donc, de savoir quelle somme économiser pour ses soins de santé. Et ils peuvent être faramineux. Par contre, pour un très large groupe d'individus, l'analyse probabiliste permet bien plus aisément de savoir combien d'argent devrait être mis en commun (à propos de cette logique assurantielle, voir Heath 20II). Cette forme de coopération n'engendre pas que des gagnants, et les "perdants» - voire ceux qui se perçoivent comme tels - ont toujours un incitatif à tenter d'éviter cette obligation.

Ces trois formes de coopération sont au cœur de l'importante interdépendance qui fait la société. En comparaison, l'interdépendance internationale se limite à des formes relativement simples de coordination et à quelques rares cas de coopération. La redistribution s'y déploie sous des formes qui valent à peine d'être soulignées. Nous sommes donc encore très loin de pouvoir développer des institutions de coopération - et donc des obligations de justice - aussi exigeantes à l'échelle globale qu'à l'échelle nationale. Dans ce contexte, l'inexistence à l'échelle globale d'un État de droit n'est pas qu'un détail. La plupart des formes de coopération qui existent à l'échelle nationale en dépendent ultimement.

La présence à l'échelle globale de nombreux problèmes d'action collective est en fait un indice important d'une coopération - et d'une interdépendance - qui y est plus faible qu'à l'échelle sociale. La coopération est une solution aux problèmes d'action collective. Leur présence confirme donc l'importance de cette différence.

Il est vrai par ailleurs qu'il est urgent aujourd'hui de trouver des solutions à de nombreux problèmes d'action collective de portée globale. C’est 
notamment le cas en matière fiscale, où il est impératif de trouver des mécanismes de coopération internationale pour faire face aux différentes formes d'échappatoire fiscal - aussi bien légal qu'illégal. C'est toutefois bien différent que de proposer d'appliquer à l'échelle globale des principes de justice distributive égalitariste.

En conclusion, comme nous le dit Philip Pettit, il ne faut jamais perdre de vue que si les théories normatives doivent être en mesure d'identifier les types d'institutions sociales qui ont le plus de chance d'être reconnues comme étant désirables, elles doivent aussi pouvoir démontrer que l'implantation de ces institutions est possible, que celles-ci peuvent être introduites et maintenues parmi des hommes et des femmes ordinaires, et non seulement parmi des saints. Les institutions doivent donc être compatibles avec notre discours normatif, mais aussi avec les incitatifs nécessaires à leur bon fonctionnement (voir Pettit, 2002, 275-276). C'est dans cet esprit que nous avons écrit La juste part.

\section{Bibliographie}

Executive Excess 2013: Bailed out, Booted, Busted. A 20 Years Review of America's Top Paid CEOs, Washington DC, Institute for Policy Studies, $43 \mathrm{p}$.

Bebchuk \& Fried. 2004. Pay Without Performance. The Unfulfilled Promise of Executive Compensation, Cambridge, Harvard University Press, 2006, 304 p.

Chavel, Solange. "La juste part peut-elle s'arrêter aux frontières? ", Philosophiques, Printemps 20I4.

Dietsch, Peter. "Ex ante ou ex post. Comment l'éducation et la formation professionnelle modifient-elles la juste part? ", Philosophiques, Printemps 20 I4.

Frank, Robert H \& Philip Cook. The Winner-Take-All Society: Why the Few at the Top Get So Much More Than the Rest of Us, New York, Penguin Books, I996, 288 p.

Heath, Joseph. "Three Normative Models of the Welfare State ", Public Reason, 3, 2OII, p. I3-44.

Hodgson, Louis-Philippe. "Why the Basic Structure? ", Canadian Journal of Philosophy, vol. 42, $\mathrm{n}^{\circ} 3 / 4,20 \mathrm{I} 2$, p. 303-334.

Néron, Pierre-Yves. "L'égalité instrumentale? Un commentaire sur La juste part», Philosophiques, Printemps 2014.

Pettit, Philip. Rules, Reasons, and Norms. Selected Essays, Oxford, Oxford University Press, 2002.

Pink, Daniel H. Drive: The Surprising Truth About What Motivates Us, New York, Riverhead Trade, 20I I, $272 \mathrm{p}$.

Rawls, John. Théorie de la justice, Paris, Seuil, I997.

Robichaud, David et Patrick Turmel. La juste part. Repenser les inégalités, la richesse et la fabrication des grille-pains, Montréal, Atelier ıо, 2012.

Sangiovanni, Andrea. "Justice and the Priority of Politics to Morality ", The Journal of Political Philosophy, vol. I6, n², 2008, p. I37-164.

Wilkinson, Richard et Kate Pickett. The Spirit Level. Why Equality is Better for Everyone, Penguin, 2009. 